\title{
自由基反应动力学模拟新策略
}

方维海

北京师范大学化学学院, 北京 100875

\section{New Strategy for Simulation of Radical Reaction Kinetics}

FANG Weihai

College of Chemistry, Beijing Normal University, Beijing 100875, P. R. China.

Email: Fangwh@bnu.edu.cn.

Published online: March 5, 2019.

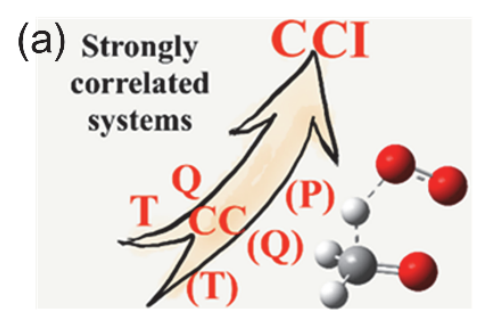

(b1)

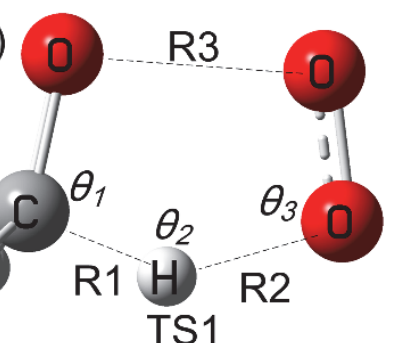

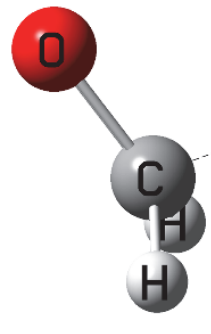

C
H)

(b2)

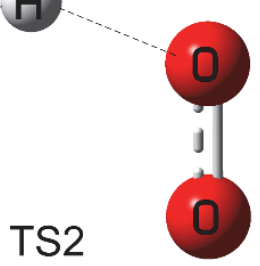

(a)示意耦合簇计算; (b) $\mathrm{CH}_{3} \mathrm{O}+\mathrm{O}_{2}$ 反应的过渡态。

自由基参与的反应动力学过程, 实验上难以 探测, 理论计算模拟也面临诸多的挑战。最近贵州 民族大学龙波教授与美国明尼苏达大学 Truhlar 课题组合作, 巧妙设计了电子结构计算策略, 定量 准确地模拟了 $\mathrm{CH}_{3} \mathrm{O}$ 自由基和三重态 $\mathrm{O}_{2}$ 分子的反 应途径和动力学, 相关结果发表在Journal of the American Chemical Society 上 ${ }^{1}$ 。作者首先用精确的 $\mathrm{CCSD}(\mathrm{T})-\mathrm{F} 12$ 电子结构方法, 结合不同的基组, 优 化反应的驻点结构, 计算振动频率并得到零点能。 计算结果表明: 用jun-cc-pVTZ或cc-pVTZ-F12基 组的 CCSD(T)-F12a计算, 能够获得可靠的几何结 构和零点能。为了得到化学精度的反应能垒, 作者 将非限制的单、双、三和四重激发和非迭代的五重 激发的耦合簇方法与完备的极限基组相结合, 设 计了 CCSDTQ $(\mathrm{P}) / \mathrm{CBS}$ 计算策略, 称之为 $\operatorname{GMM}(\mathrm{P})$ 方
法。该方法计算精度接近FCI水平。在仔细分析计 算的自由基反应能垒的基础上, 发现其中的四重 激发起到了关键作用。随后作者们应用包括小曲 率隧道效应的正则变分过渡态理论, 计算了不同 温度下自由基反应的速率常数, 确定Arrhenius活化 能随温度的变化关系。

作者设计的计算策略不仅能够获得反应体系 的热力学参数, 而且可以得到不同温度和压力下 的准确反应速率常数。一方面, 该工作为进一步的 实验研究提供了可靠的参考; 另一方面, 设计的新 策略可用于研究其它自由基反应的动力学。

\section{References}

(1) Long, B.; Bao, J. L.; Truhlar, D. G. J. Am. Chem. Soc. 2019, 141, 611. doi: $10.1021 /$ jacs.8b11766 